\title{
Bone and joint changes in lower limb amputees
}

\author{
M. J. BURKE, * V. ROMAN, AND V. WRIGHT
}

From the Rheumatism Research Unit, University Department of Medicine, General Infirmary at Leeds, and DHSS Artificial Limb and Appliance Centre, Chapel Allerton, Leeds

SUMMARY A clinical and radiological survery of bone and joint changes in 42 lower limb.amputee्s is reported. There was a significant increase in osteoarthrosis in the knee of the unamputated le⿳亠丷厂⿰氵亏 compared with the amputated side. The amputated side characteristically showed osteoporosis Comparing the prevalence of osteoarthrosis in this study with figures in the population, it is mote likely that the osteoporosis of the amputated limb had a protective effect on that side than that mechanical factors produced more osteoarthrosis on the contralateral side. Backache occurred in about half the patients, and was severe in $19 \%$. It did not appear to be related to disc degeneration, but scoliosis was observed in $64 \%$ of patients.

As part of an extensive investigation into clinical and biomechanical aspects of osteoarthrosis, groups of subjects who might be especially at risk have been studied at Leeds. These include professional soccer players (Adams, 1976), sports and ex-military parachutists (Murray-Leslie et al., 1977), pneumatic drillers (Burke et al., 1977), and physical education teachers (C. J. Eastmond, C. Hudson and V. Wright, unpublished data). Another group who might be expected to develop changes due to disordered biomechanics are patients with a limb amputated who have been fitted with a prosthesis. We report here the results of a survey carried out in a group of lower limb amputees, attending the Chapel Allerton Artificial Limb and Appliance Centre.

\section{Method}

Forty-two unselected single-leg amputees (38 men, 4 women) took part in the study. All were under the age of 65 and had worn a prosthesis for at least 5 years. Inquiry was made into the reasons for amputation, level of amputation, history of other injuries to the legs, and presence of rheumatic symptoms. In the examination a detailed evaluation was made of the legs and lumbar spine, noting swelling, tenderness, limitation of movement, and in the lumbar spine, scoliosis.

Accepted for publication September 5, 1977

Correspondence to Professor V. Wright, Rheumatism Research Unit, School of Medicine, 36 Clarendon Road, Leeds LS2 9PJ

*Present address: Department of Rheumatology, Manchester Royal Infirmary.
$X$-rays were taken of the lumbar spine (anter $\vec{\xi}$ posterior and lateral), pelvis, and knee(s). They wefe graded for osteoarthrosis by two observers (M.J.8. and V.W.), using standard criteria (Jeffrey et ak 1963), nil 0, doubtful 1, mild 2, moderate 3 , severe Osteoporosis, scoliosis, and other bony changes wees noted.

\section{Results}

The amputees' mean age was $48 \cdot 4$ years (range $27-6 \overline{4}$ years) with an average duration from the time of amputation of $24 \cdot 6$ years (range 7-51 years). The mean weight was $70.3 \mathrm{~kg}$. Amputation had beefl performed for trauma in 34 subjects, osteomyelit in 5 , and tumour in 3 . None had a history of injuriog to the intact leg. The level of amputation was above the knee in 19, below the knee in 22 , and through hip in one.

\section{SYMPTOMS}

None of the below-knee amputees had symptonge from the knee of the amputated leg. 22 of the 42 amputees had symptoms from the unaffected opposite knee, considered minimal in 7 , mild in $\&$ and of moderate severity in 7. 3 amputees has minimal symptoms in the hip on the amputated side while 7 had symptoms in the opposite hip, minima in 5 , mild in 2 and moderate in 3 . Backache occurref in 20 amputees being of moderate or severe intensitw in 8 .

\section{PHYSICAL SIGNS}

Minor limitation of hip movement was present in amputees; in 2 of these it was bilateral. The hip o 
the amputated leg alone was limited in 2 and the ipsilateral hip in one. In the knee of the unaffected leg crepitus on movement was present in 11 subjects. One knee effusion was noted. 2 amputees had flexion deformities (of $15^{\circ}$ and $20^{\circ}$ in the knee). Scoliosis was found in 27 amputees.

\section{RADIOLOGY}

The radiological gradings for osteoarthrosis are given in the Table. None of the amputees had grade 4 osteoarthrosis in the hips or knee. 7 amputees $(16.7 \%$ of the total) had radiological evidence of osteoarthrosis (grade 2 or 3 ) in the knee of the unamputated leg, while none of the other below-knee amputees had osteoarthrosis in that knee. The difference is probably significant $(P<0.05)$. There was a slight but not significant increase in osteoarthrosis in the hip of the unamputated leg compared with the hip on the amputated side. The mean age of patients with osteoarthrosis in the knees was 56.5 years (range 53-63 years), with osteoarthrosis of the hip 44.6 years (range 37-57), and with osteoarthrosis of either hip or knee 49.7 years. Osteoporosis in the amputated limb was common, being noted in 37 amputees $(88 \%)$. It was marked in 5 cases where there was hemiatrophy of the pelvis.

Scoliosis was noted on $x$-ray in 18 amputees $(42.9 \%)$. Degenerative changes were present in the lumbar spine in 32 amputees $(76 \%)$. The detailed changes were: at D12/L1 1 had grade 2, 3 had grade 3 ; at L1/2 7 had grade 2, 2 grade 3; at L2/3 9 had grade 2, 1 grade 3; at L3/4 9 had grade 2; at L4/5 6 had grade 2, 1 grade 4; at L5/S1 2 had grade 2, 1 grade 3,3 grade 4 .

Other changes found were occult spina bifida in 3 and abnormal sacroiliac joints in 2 cases. One had bilateral sacroiliitis and the other patient who had a right below-knee amputation showed sclerosis of the left sacroiliac joint.

\section{Discussion}

Our findings are in keeping with those of Hunger-

Table Radiological grading for osteoarthrosis

\begin{tabular}{lllll}
\hline & Grade & & \\
\cline { 2 - 5 } & 1 & 2 & 3 & $\begin{array}{l}\text { Total } \\
\text { grades } 2 \\
\text { and 3 }\end{array}$ \\
\hline Knee & & & & \\
$\quad$ Amputated side & 5 & 0 & 0 & $0 / 22$ \\
$\quad$ Unamputated leg & 4 & 6 & 1 & $7 / 42$ \\
Patellofemoral & & 0 & 0 & $0 / 22$ \\
$\quad$ Amputated side & 0 & 0 & 1 & $1 / 42$ \\
$\quad$ Unamputated leg & 3 & 0 & $2 / 41$ \\
Hip Amputated leg & 2 & 2 & 0 & $5 / 42$ \\
$\quad$ Unamputated leg & 3 & 3 & 2 & \\
\hline
\end{tabular}

ford and Cockin (1974) who in a survey of 63 belowknee and 54 above-knee World War II single-leg amputees found a significant increase in tibiofemoral osteoarthrosis on the unamputated side. Osteoporosis was present on the amputated side. They also found a marked increase in patellofemoral osteoarthrosis in the unamputated leg, but this was not apparent in our study.

Analysis of above-knee amputee gait (Eberhart et al., 1954) using force-plate records has shown that the unamputated leg carries more load than the prosthetic leg. Moreover, the time spent on the artificial leg is markedly less than that spent on the natural leg. This was the explanation Borgmann (1959) gave for the development of osteoarthrosis in the hips and knees of amputees. Inequality of leg length may also contribute to increased stress on the intact leg (Dixon and Campbell-Smith, 1969); to facilitate the forward swing phase in walking the prosthetic leg is made slightly shorter than the unamputated leg.

These factors, all of a mechanical nature, could be the cause of an increased prevalence of osteoarthrosis. However, it should be noted that none of the changes were severe, and indeed the majority were mild. In other groups, notably professional soccer players and pneumatic drillers, we noted 'osteophytic' changes, that would often be interpreted as osteoarthrosis, without joint-space narrowing. We have suggested that these are not really the changes of osteoarthrosis but the reaction to stress in ligaments and tendons adjacent to the joint.

The characteristic osteoporosis seen in the amputated limb almost certainly represents a form of disuse artrophy. It may well have a protective effect, accounting for the lower prevalence of osteoarthrosis in the joints of the amputated limb. Comparison of our figures with data from a similar age group in the population (Lawrence, 1969) suggests that this is the more likely explanation. It is in keeping with the findings of Glyn et al. (1966) of a low prevalence of osteoarthrosis in hip and knee in a late review of patients with anterior poliomyelitis. These authors attributed the decreased prevalence to fewer mechanical stresses, but it could equally have been due to the better shock absorption by osteoporotic bone, as suggested by Radin (1976).

Backache was frequent and occurred more commonly than in the general population (Lawrence and Aitken-Swan, 1952). However, disc degeneration was no more frequent (Lawrence, 1969). The presence of scoliosis in $64 \%$ of the amputees suggests that mechanical factors consequent on the amputation may be responsible for the back symptoms. 


\section{References}

Adams, I. D. (1976). Osteoarthrosis and sport. Clinics in Rheumatic Diseases, pp. 523-541. Ed. by V. Wright. Saunders, London.

Borgmann, F. (1960). Zur gutachtlichen Beurteilung von Ruckenbeschwerden und befunden bei Oberschelamputation. Zeitschrift für Orthopädie und ihre Grenzgebeite, 93, 351-364.

Burke, M. J., Fear, E. C., and Wright, V. (1977). Bone and joint changes in pneumatic drillers. Annals of the Rheumatic Diseases, 36, 276-279.

Dixon, A. St. J., and Campbell-Smith, S. (1969). Long leg arthropathy. Annals of the Rheumatic Diseases, 28, 359-365.

Eberhart, H. D., Elftman, H., and Inman, V. T. (1954). The locomotor mechanism of the amputee. Human Limbs and their Substitutes. McGraw-Hill, New York.

Glyn, J. H., Sutherland, I., Walker, G. F., and Young, A. C. (1966). Low incidence of osteoarthrosis in hip and knee after anterior poliomyelitis: a late review. British Medical Journal, 2, 739-742.
Hungerford, D. S., and Cockin, J. (1974). The fate of the retained lower limb joints in World War II amputêes. Abstract, British Orthopaedic Association Autumn Meeting.

ज़

Jeffrey, M. R., Kellgren, J. H., and Ball, J. R. (1963). ॠhe Epidemiology of Chronic Rheumatism, Vol. 2. Atla $\delta 0 f$ Siandard Radiographs of Arthritis. Blackwell, Oxford.

Lawrence, J. S. (1969). The epidemiology of rheum diseases. Textbook of Rheumatic Diseases, 4th ed., $\Phi$ p. 163-181. Ed. by W. S. C. Copeman. Livingstone, Eginburgh.

Lawrence, J. S., and Aitken-Swan, J. (1952). RheumatisrPin miners. 1. Rheumatic complaints. British Journal Industrial Medicine, 9, 1-12.

Murray-Leslie, C. F., Lintott, D. J., and Wright, V. (19: The knees and ankles in sport and veteran miliary parachutists. Annals of the Rheumatic Diseases, 36 , 327-331.

Radin, E. L. (1976). Aetiology of osteoarthrosis. Clinicisu in Rheumatic Diseases, pp. 509-521. Ed. by V. Wright. Saunders, London. 